\title{
POTENCIAIS OPORTUNIDADES FORMATIVAS COM MTSK E PESQUISAS CIENTÍFICAS SOBRE FRAÇÕES E OPERAÇÕES
}

\author{
POTENTIAL TRAINING OPPORTUNITIES WITH MTSK AND SCIENTIFIC \\ RESEARCH ON FRACTIONS AND OPERATIONS
}

\section{POSIBLES OPORTUNIDADES FORMATIVAS CON MTSK E INVESTIGACIÓN CIENTÍFICA SOBRE FRACCIONES Y OPERACIONES}

\author{
Jeferson Gomes Moriel Junior $^{1}$ (iD) $(2)$ \\ Gladys Denise Wielewski ${ }^{2}$ (D)
}

\begin{abstract}
RESUMO
As pesquisas com o Mathematics Teachers' Specialized Knowledge (MTSK) têm crescido no mundo e no Brasil, entretanto ainda há poucos estudos sobre criação e avaliação de atividades formativas com tal marco teórico. O objetivo deste artigo é discutir teoricamente como a combinação das dimensões do MTSK com resultados científicos em Educação Matemática pode oferecer caminhos formativos para potencializar o desenvolvimento de conhecimento especializado docente sobre frações e operações, seu ensino e aprendizagem. Trata-se de uma pesquisa qualitativa analítico-descritiva, de caráter teóricoexploratório, em que analisamos um trecho de uma oficina formativa na qual um licenciando foi convidado a refletir sobre a sequenciação didática para ensinar os referidos conteúdos e a partir da sua resposta realizamos uma análise reflexiva a posteriori sobre caminhos formativos possíveis. Nossos resultados mostram tais caminhos, ligados a todas as dimensões MTSK fundadas em resultados científicos, dentre os quais destacamos: (i) recomendações de encadeamento de conceitos sobre frações e operações provenientes de especificações curriculares, pesquisas científicas ou opinião de professores experientes, elaboradas levando em consideração as características de aprendizagem de estudantes, erros comuns e suas fontes prováveis; (ii) conceitos, algoritmos, procedimentos, justificativas e fenômeno ligados a frações e suas operações, bem como, diferentes conexões entre tais conceitos; (iii) explicações instrucionais e estratégias didáticas que permitam ensinar para a compreensão por meio do fazer matemática, ao invés de apenas memorizá-la, incluindo conhecimentos da prática de definir e demonstrar.
\end{abstract}

Palavras-chave: Oportunidades formativas. MTSK. Formação docente. Educação matemática. Frações.

\section{ABSTRACT}

Research with Mathematics Teachers' Specialized Knowledge - MTSK has grown in the world and in Brazil, although there are still few studies about creation and evaluation of training activities with such a theoretical framework. The purpose of this article is to discuss theoretically how a combination of the

\footnotetext{
${ }^{1}$ Doutor em Educação em Ciências e Matemática pela Universidade Federal de Mato Grosso (UFMT-REAMEC). Professor do Instituto Federal de Mato Grosso (IFMT) e do Programa de Pós-graduação em Ensino do IFMT/UNIC, campus Cuiabá, Mato Grosso, Brasil. Endereço para correspondência: Rua das Brisas, 45, apto 1205, Torre 1, Despraiado, Cuiabá, Mato Grosso, Brasil, CEP: 78048-225. E-mail: jeferson.moriel@cba.ifmt.edu.br.

${ }^{2}$ Doutora em Educação Matemática pela Pontifícia Universidade Católica de São Paulo (PUC). Professora do Depto. de Matemática e do Doutorado em Educação em Ciências e Matemática (REAMEC) da Universidade Federal de Mato Grosso (UFMT), Cuiabá, MT, Brasil. Endereço para correspondência: Av. Fernando Corrêa da Costa, 2367, Boa Esperança, Cuiabá, MT, Brasil, CEP: 78060-900. E-mail: gladysdw@gmail.com
} 
dimensions of the MTSK with scientific results in Mathematical Education can offer training sources to enhance the development of specialized teaching knowledge about fractions and operations, their teaching and learning. It is a qualitative analytical-descriptive research, of a theoretical-exploratory nature, in which we analyzed an excerpt from a training workshop in which a graduate was invited to reflect on the didactic sequencing to use the contents and based on his answer, we conducted a a posteriori reflective analysis on possible training paths. Our results show such paths, linked to everyone as MTSK dimensions based on scientific results, among which we highlight: (i) recommendations for linking concepts about fractions and procedures related to curricular specifications, scientific research or the opinion of experienced teachers, developed taking into account student learning characteristics, common mistakes and their likely sources; (ii) concepts, algorithms, procedures, justifications and phenomena related to fractions and their operations, as well as, different among such concepts; (iii) instructional and didactic clinical explanations that they use to understand through exercise, doing math, just memorizing it, including knowledge of the practice of defining and demonstrating.

Keywords: Formative opportunities. MTSK. Teacher training. Mathematics education. Fractions.

\section{RESUMEN}

Investigación con Mathematics Teachers' Specialized Knowledge - MTSK ha crecido en el mundo y en Brasil, sin embargo, aún existen pocos estudios sobre la creación y evaluación de actividades de formación con este marco teórico. El objetivo de este artículo es discutir teóricamente cómo la combinación de las dimensiones del MTSK con resultados científicos en Educación Matemática puede ofrecer vías de formación para potenciar el desarrollo de conocimientos docentes especializados sobre fracciones y operaciones, su enseñanza y aprendizaje. Se trata de una investigación cualitativa analíticodescriptiva, de carácter teórico-exploratorio, en la que se analizó un apartado de un taller de formación en el que se invitó a un egresado a reflexionar sobre la secuencia didáctica para enseñar los contenidos referidos y en base a su respuesta un análisis reflexivo a posteriori sobre posibles caminos formativos. Nuestros resultados muestran tales caminos vinculados a todas las dimensiones del MTSK con base en resultados científicos, entre los que destacamos: (i) recomendaciones para vincular conceptos sobre fracciones y operaciones a partir de especificaciones curriculares, investigación científica o la opinión de profesores experimentados, elaborada teniendo en cuenta las características de aprendizaje de los estudiantes, los errores comunes y sus probables fuentes; (ii) conceptos, algoritmos, procedimientos, justificaciones y fenómenos vinculados a las fracciones y sus operaciones, así como las diferentes conexiones entre dichos conceptos; (iii) explicaciones instruccionales y estrategias didácticas que permitan enseñar para la comprensión a través de la práctica matemática, en lugar de solo memorizarla, incluyendo el conocimiento de la práctica de definir y demostrar.

Palabras clave: Oportunidades formativas. MTSK. Formación de profesores. Educación Matemática. Fracciones.

\section{INTRODUÇÃO}

No Brasil, uma quantidade pequena de estudantes têm aprendido o que deveria em termos de matemática da educação básica nos último 20 anos (INEP, 2014; 2017). Existem diversos aspectos que podem melhorar os índices de aprendizagem, a exemplo de avanços na formação docente. Esforços neste sentido têm sido feitos a partir de pesquisas científicas baseadas em diferentes tipologias do conhecimento docente necessário para ensinar matemática (SHULMAN, 1986; TARDIF, 2002; BALL; THAMES; PHELPS, 2008; ROWLAND, 2013). Dentre elas, tem crescido muito, particularmente no Brasil (MORIEL JUNIOR; DUARTE, 
2020), as pesquisas que usam as seis dimensões do Mathematics Teachers' Specialized Knowledge - MTSK (CARRILLO et al., 2014; CARRILLO-YAÑEZ et al., 2018).

O MTSK é o marco teórico mais recente na área e supera limitações de modelos anteriores (MONTES; CONTRERAS; CARRILLO, 2013; KILPATRICK; SPANGLER, 2015). As pesquisas com MTSK tem resultado na configuração de panoramas completos e interconectados de conhecimentos mobilizados para ensinar e fazer aprender determinados conteúdos, a exemplo de frações e geometria (MORIEL JUNIOR, 2014; ROJAS, 2014; AGUILAR, 2016). Entretanto, ainda há poucos estudos sobre como promover a construção de tais conhecimentos especializados em licenciandos e professores de Matemática e sobre tarefas formativas (RIBEIRO, 2016; GARCIA; PRIETO; CATALÁN, 2017; MORIEL JUNIOR et al., 2017; QUIROGA; GAMBOA, 2017; CLIMENT; MONTES, 2019; LIZARDE, 2019).

Neste cenário, entendemos que se encontram abertas na área algumas perguntas importantes: Como um formador pode promover intencionalmente em um (futuro) professor a construção de conhecimento especializado em determinada dimensão do MTSK ou mesmo em todas elas? Como realizar essa formação docente fundada em bases sólidas de resultados científicos conectados à prática docente? Como isso pode ser articulado com conhecimentos ou crenças que o (futuro) docente possui ou não, de modo a construir, aumentar ou aprofundar seu conhecimento especializado?

Certos da complexidade envolvida na construção de respostas a essas perguntas, optamos por iniciar a exploração deste cenário por meio de uma aproximação teórica, para em estudo posterior abordar empiricamente o assunto. Por isso, o objetivo deste artigo é discutir teoricamente como a combinação das dimensões do MTSK com resultados científicos em Educação Matemática pode oferecer caminhos formativos para potencializar o desenvolvimento de conhecimento especializado docente sobre frações e operações, seu ensino e aprendizagem.

Para tanto, analisamos um episódio empírico extraído de uma oficina formativa pertencente a um estudo mais amplo (MORIEL JUNIOR, 2014) - em que um licenciando foi convidado a refletir sobre a sequenciação didática para ensinar os referidos conteúdos. A partir da sua resposta realizamos uma análise reflexiva a posteriori sobre caminhos formativos possíveis utilizando a estrutura do MTSK e resultados científicos na área, sem a pretensão de esgotá-los quantitativamente, mas sim fornecer um panorama dentre os possíveis. 


\section{REFERÊNCIAL TEÓRICO}

O Mathematics Teachers' Specialized Knowledge - MTSK é um modelo teórico que descreve o conhecimento específico e especializado que um professor pode (ou deve) ter para ensinar Matemática. Ele é composto por dois domínios (o Conhecimento Matemático e o Conhecimento Didático do Conteúdo) e cada um deles possui três subdomínios, representados pelo hexágono, conforme Figura 1. Cada subdomínio engloba um conjunto de categorias e indicadores particulares de conhecimentos que compõem a base de conhecimento de professores que ensinam Matemática (CARRILLO et al., 2014; CARRILLO et al., 2017; CONTRERAS et al., 2017; CARRILLO-YAÑEZ et al., 2018). No centro do modelo, encontram-se as crenças sobre a Matemática, sobre o ensino e a aprendizagem dessa disciplina que dão sentido ao trabalho dos professores em questão.

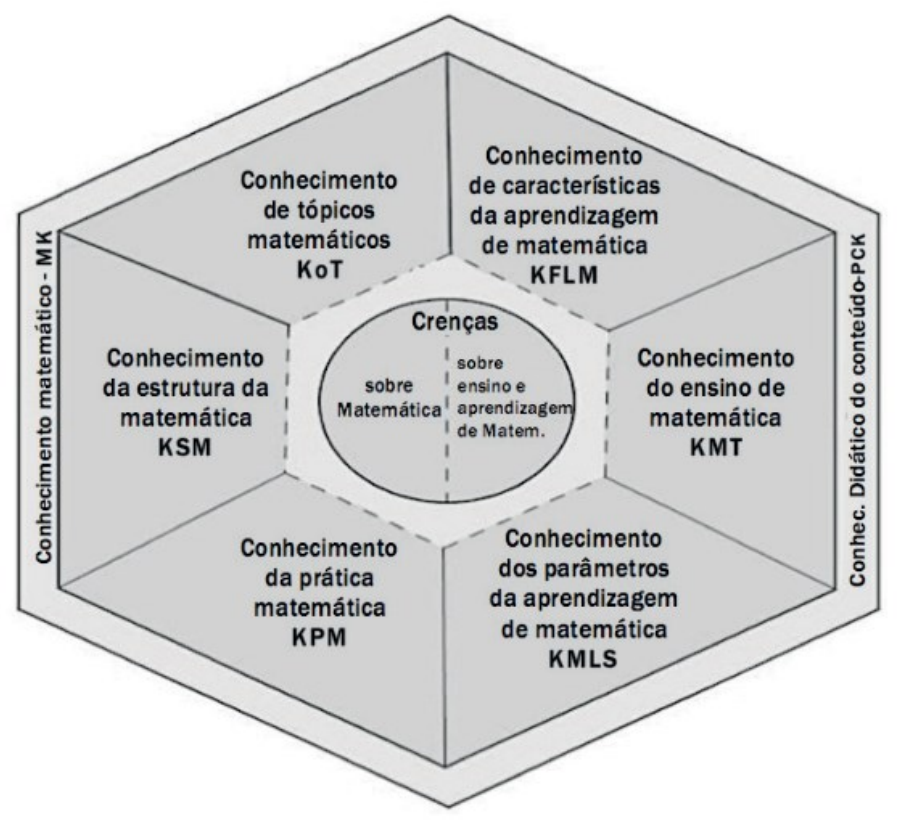

Figura 1 - Domínios e subdomínios do modelo MTSK $^{3}$

Fonte: Carrilo et al. (2014) traduzido por Moriel Junior e Wielewski (2017, p. 130).

Os subdomínios do Conhecimento Matemático são: (i) Conhecimento dos Tópicos (KoT), que engloba os tópicos da Matemática isoladamente, além de procedimentos, definições, propriedades, aplicações e fenômenos, bem como as diferentes representações de um conteúdo; (ii) Conhecimento da Estrutura da Matemática (KSM), que se refere às conexões conceituais entre tópicos das diferentes áreas da Matemática, ou seja, esse subdomínio reporta as relações

\footnotetext{
${ }^{3}$ Utilizamos as siglas oriundas do idioma inglês para manter a padronização internacional do modelo.
} 
entre os tópicos elementares e avançados, prévios e futuros, abrangendo também o conhecimento das situações nas quais haja similaridade de pensamento entre conteúdos de distintas áreas; (iii) Conhecimento da Pratica da Matemática (KPM), que inclui os conhecimentos sobre modos de produzir em Matemática, elementos que estruturam uma demonstração e estratégias para argumentar, generalizar e explorar matematicamente.

Os subdomínios do Conhecimento Didático do Conteúdo são: (i) Conhecimento do Ensino de Matemática (KMT), que inclui teorias de ensino (formais e pessoais), estratégias e atividades de ensino, tais como as tendências em educação da Matemática, abrange o conhecimento de explicações instrucionais e os diversos modos e recursos para apresentar um conteúdo matemático; (ii) Conhecimento das Características da Aprendizagem de Matemática (KFLM), que são os conhecimentos sobre como os alunos aprendem os conteúdos matemáticos, a forma de interagir com os conteúdos (como estratégias comuns de resolução de problemas), as características do processo de compreensão, os erros comuns, as dificuldades e a linguagem comumente usada em sala de aula pelos aprendizes ao lidarem com o conteúdo, bem como, teorias (formais e pessoais) sobre o desenvolvimento cognitivo dos alunos em relação à Matemática; (iii) Conhecimento do Parâmetros da Aprendizagem de Matemática (KMLS), que se refere às expectativas de desenvolvimento conceitual e procedimental esperado em cada etapa escolar, assim como os conteúdos a serem ensinados. São fontes desse conhecimento as especificações curriculares, as normas mínimas, as formas de avaliação para progressão do aluno e os objetivos de desempenho de organismos externos.

Os domínios, subdomínios e as categorias do MTSK são utilizados por investigadores como instrumento de análise de dados para caracterizar conhecimentos mobilizados por (futuros) professores nas diversas etapas que envolvem o ensino de Matemática. Eles têm sido utilizados como pano de fundo de livros internacionais, ainda não traduzidos para o português, destinados à formação de professores (CARRILLO et al., 2016; CATALÁN; CARRILLO, 2018). Neste artigo, eles serão utilizados para discutirmos teoricamente possibilidades formativas, ou seja, como um formador de professores pode utilizar o MTSK para desenvolver o conhecimento especializado de docentes e licenciandos.

\section{ENCAMINHAMENTO METODOLÓGICO}

Trata-se de uma pesquisa qualitativa analítico-descritiva (BOGDAN; BIKLEN, 1991), teórico-exploratória sobre possibilidades formativas baseadas em conhecimento especializado 
para ensinar e fazer aprender Matemática. O contexto da pesquisa é um recorte de uma oficina formativa que os autores realizaram para professores e licenciandos em Matemática sobre frações e suas operações dentro do Projeto "Observatório da Educação" (OBEDUC / UFMT, Cuiabá). Este recorte consiste em uma parte dos dados empíricos obtidos para uma pesquisa doutoral mais ampla (MORIEL JUNIOR, 2014), utilizando como ponto de partida a manifestação de um licenciando em Matemática que estava nas etapas finais do curso e tinha realizado estágios obrigatórios e participado do referido Projeto em escolas da educação básica e trabalhado os conteúdos discutidos na formação.

A escolha do sujeito e do episódio para este artigo se baseou nos seguintes critérios: (i) ter foco em alguma questão relevante e aparentemente simples da prática docente (no caso, sobre sequenciação de conteúdos e diretrizes curriculares) e (ii) o sujeito manifestar pouco ou nenhum conhecimento durante a resposta questão.

Desta forma, obtivemos um episódio de um sujeito que nos permitiu analisar um caso com maior potencial para nosso objetivo de discutir caminhos formativos possíveis para aquela situação. Adicionalmente, aqueles critérios refletem um cenário relativamente comum em formação inicial ou continuada, que é a existência de lacunas e limitações de conhecimento docente em relação a aspectos elementares do conteúdo, seu ensino ou aprendizagem (NAISER; WRIGHT; CAPRARO, 2003; PINILLA, 2007; BERTONI, 2008; BAYOUD, 2011; FÁVERO; PINA NEVES, 2012).

A obtenção de dados ocorreu por meio de gravação audiovisual da oficina formativa e transcrição, a partir da qual fizemos leituras sucessivas do material e extraímos um episódio considerando as características e critérios anteriores. Isto resultou em um trecho da manifestação de um licenciando sobre a sequenciação de conteúdos e diretrizes curriculares, para que fosse possível a análise das evidências de conhecimento especializado mobilizado e para a discussão das possibilidades formativas associadas a cada subdomínio MTSK.

A análise de conhecimentos foi feita por meio de comparações sistemáticas entre as unidades de análise (trechos das transcrições) e as definições das categorias dos subdomínios MTSK (CARRILLO et al., 2014). A partir desses elementos, realizamos uma análise reflexiva a posteriori baseada na estrutura do MTSK e de resultados de pesquisas científicas dentro da temática (a partir de mapeamentos da área e bases de dados) para identificar caminhos e oportunidades formativas que poderiam (ou podem) ajudar a desenvolver (mais) o conhecimento especializado do sujeito (e de outros em situação similar). Não pretendemos ou achamos que seja possível esgotar todas as possibilidades de caminhos, por isso aqui se trata 
de um panorama dentre os possíveis. Assim, focalizamos a contribuição do MTSK como instrumento de reflexão ao formador de professores interessado em potencializar suas ações.

\section{RESULTADOS E DISCUSSÃO}

Em determinado momento da oficina formativa, após discutirmos algoritmos da divisão de frações (MORIEL JUNIOR; WIELEWSKI, 2013b; MORIEL JUNIOR; WIELEWSKI; CARRILLO, 2019) e que foram relacionados com os da adição e multiplicação, o pesquisador colocou a pergunta sobre qual seria a ordem didática adequada para ensinar tais operações e qual seria a justificativa para isso. Trata-se de um questionamento aparentemente simples e de alta importância à tarefa docente, endereçado predominantemente para uma questão curricular associada ao conhecimento dos parâmetros da aprendizagem Matemática (KMLS). O licenciando até ali houvera manifestado conhecimento procedimental (no subdomínio dos tópicos KoT, categoria como fazer) dos algoritmos das referidas operações, mas neste momento mencionou não saber qual delas (adição ou multiplicação de frações) é ou deveria ser ensinada primeiro na educação básica, deixando subentendido que a divisão seria posteriormente.

\footnotetext{
Licenciando: Qual é a regra que vem antes [adição ou multiplicação]? Eu não sei qual que ensina primeiro. [...] Didaticamente, eu não sei qual que é melhor ensinar primeiro. [...] Eu acho que seria melhor ensinar primeiro a adição, porque ensinando o mais difícil primeiro (vamos supor que o aluno não saiba nada, não saiba a regra da multiplicação) e colocando muitas situações para ele somar, com denominadores diferentes, para ele se habituar bem com a regra, quando você ensinar a regra da multiplicação vai ser muito mais fácil pra ele aprender. Aí ele não vai querer aplicar essa regra aqui [da multiplicação], porque ele já aprendeu isso aqui [o procedimento da adição]. Estou indo pela lógica, mas não sei qual que ensina primeiro.
}

(Trecho do diálogo entre pesquisador e licenciando, 2013)

$\mathrm{Na}$ falta de conhecimento proveniente de especificações curriculares, pesquisas científicas ou opinião de professores experientes (enquadrado no subdomínio dos parâmetros da aprendizagem matemática, KMLS) o licenciando não teve outra alternativa senão adotar um critério que ele considerou mais plausível logicamente (“começar pelo mais difícil”) para decidir sobre o encadeamento dos conteúdos, sobre qual operação de frações ensinar primeiro. Entendemos que neste caso, o licenciando fez um movimento cognitivo em direção ao centrodireita do MTSK ao mobilizar crenças sobre ensino e sobre aprendizagem de matemática ("vai ser muito mais fácil pra ele aprender"), ao invés de conhecimentos especializados.

O episódio em questão tem o conhecimento dos parâmetros de aprendizagem Matemática (KMLS) como central. Desta forma, há uma possibilidade direta de formação para 
desenvolver conhecimento do licenciando neste subdomínio, abordando aspectos curriculares na categoria sequenciação didática de conteúdos (CARRILLO et al., 2014; CARRILLOYAÑEZ et al., 2018). Neste sentido, pode-se discutir diretrizes curriculares internacionais (WU, 2011; LIN et al., 2013) e, especialmente, a Base Nacional Comum Curricular (BRASIL, 2018). Tal documento oficial recomenda que a adição e a subtração de números racionais sejam tratadas no sexto ano do ensino fundamental (código EF06MA10) e a multiplicação e divisão no sétimo (EF07MA10). Entretanto, a complexidade da docência envolve o domínio de outros conhecimentos didáticos do conteúdo (PCK).

A situação dá margem formativa para explorar e desenvolver aspectos do subdomínio do ensino de Matemática (KMT), abordando métodos, recursos, estratégias e teorias de ensino, dentre os quais destacamos as frações e suas operações como um objeto do ensino escolar (PINILLA, 2007; CASTRO; OLIVEIRA, 2009; PETIT; LAIRD; MARSDEN, 2010), o que são boas explicações instrucionais e suas características no referido conteúdo (FORRESTER; CHINNAPPAN, 2010; CHARALAMBOUS; HILL; BALL, 2011) e tendências em Educação Matemática que podem ser utilizadas, dentre as quais destacamos Resolução de problemas, Modelagem matemática, História da matemática em sala de aula com ou sem o uso de Tecnologias da informação e comunicação (RICONSCENTE, 2011).

Detectamos uma oportunidade para explorar formativamente os resultados de pesquisas científicas sobre dificuldades de aprendizagem de alunos sobre frações e suas operações, seus erros comuns e prováveis fontes (PINILLA, 2007; LOPES, 2008; BAYOUD, 2011; SANTOS; SANTOS; CAMPOS, 2013), refletindo sobre como as opções de ensino ajudam a superá-las ou mesmo evitar que apareçam (KILPATRICK; SWAFFORD; FINDELL, 2001; ASHLOCK, 2006). Inclui-se dentre as fontes de informação neste domínio didático de conhecimento aquelas provenientes da cultura escolar, como teorias pessoais de professores experientes, e aquelas baseadas em resultados de avaliações de larga escala (simulados nas escolas ou provas desenvolvidas por órgãos externos como SAEB e PISA) e de pesquisas na área de Educação Matemática, incluindo problemas a serem superados no ensino de frações, dentre outros (PINILLA, 2007; CASTRO; OLIVEIRA, 2009; PETIT; LAIRD; MARSDEN, 2010).

Do domínio matemático (CARRILLO et al., 2014; CARRILLO-YAÑEZ et al., 2018), também há elementos que poderiam ser úteis para construir respostas à questão norteadora do episódio e que consequentemente significam oportunidades de formação para construção destes conhecimentos especializados. No subdomínio de tópicos (KoT), faz-se necessário que o professor possua não só o conhecimento procedimental sobre as operações como algoritmos e 
procedimentos alternativos (GUERRA; SILVA, 2008; BERTONI, 2009), como também o conceitual. Isto inclui diferentes significados e modos de compreender o conceito de frações (BEHR et al., 1983) e operações (MA, 1999; SILVA; ALMOULOUD, 2008; MORIEL JUNIOR, 2017), permitindo assim que o professor consiga criar diferentes contextos concretos em que os conceitos se aplicam ou foram originados (FERREIRA, 2006; LOPES, 2008; CASTRO; OLIVEIRA, 2009), ligados à categoria fenômenos e aplicações (KoT).

Destacamos que é comum professores desconhecerem as diversas interpretações de fração (também chamadas de subconstructos, personalidades, significados ou sentidos), sendo que a mais conhecida e ensinada é a ideia de "parte-todo" (NUNES; BRYANT, 1997; BERTONI, 2008; CAMPOS; SILVA, 2009). Embora a quantidade de interpretações tenha variado ao longo dos anos (chegado até 12 ideias distintas), atualmente existem cinco consideradas principais: relação parte-todo, medida, razão, quociente indicado e operador (BEHR et al., 1983; LOPES, 2008; MOREIRA; FERREIRA, 2008; DRECHMER; ANDRADE, 2011).

Ainda no subdomínio dos Tópicos (KoT), inclui-se o conhecimento dos porquês matemáticos que justificam os procedimentos e as operações, para responder por exemplo: Por que inverte-e-multiplica? Por que não pode zero no denominador? (LIMA, 1983; GUERRA; SILVA, 2008; ANGELO; DOS SANTOS; MELÃO, 2009; BARBOSA, 2011; MORIEL JUNIOR; WIELEWSKI, 2013a). Por vezes, esta justificativa vai promover conexões intraconceituais (CARRILLO et al., 2014; VASCO; MORIEL JUNIOR; CONTRERAS, 2017) que articulam matemática da educação básica e matemática da educação superior, por exemplo, divisão de frações, corpo algébrico e classes de equivalência. Isto favorece uma formação que articula teoria e prática (MOREIRA; DAVID, 2003; FIORENTINI, 2005; MORIEL JUNIOR; WIELEWSKI; CARRILLO, 2019).

Além desses conhecimentos isolados, percebemos a oportunidade formativa para desenvolver o conhecimento que conecte conceitos envolvidos com frações ou com as operações, pertencente ao subdomínio da estrutura matemática (KSM), pois isto também poderia ser balizador da escolha mais adequada para a sequência de conceitos a serem ensinados (pertencente ao KMT). Exemplo disso é que Lopes (2008) apresenta uma proposta alternativa para ensinar frações por meio da qual conecta diferentes conceitos, como frações equivalentes, comparações, adição, subtração de inteiros, aproximação, arredondamento, limites, diferentes representações e calculadora. Isto reforça o argumento de que "não é possível isolar cada uma das ideias das frações e suas interpretações, algumas delas têm vínculos naturais" (LOPES, 
2008, p. 9), como encontra-se em diversos esquemas em diversos países (BEHR et al., 1983; MA, 1999), a exemplo da rede identificada em uma formação no México, conforme Figura 2.

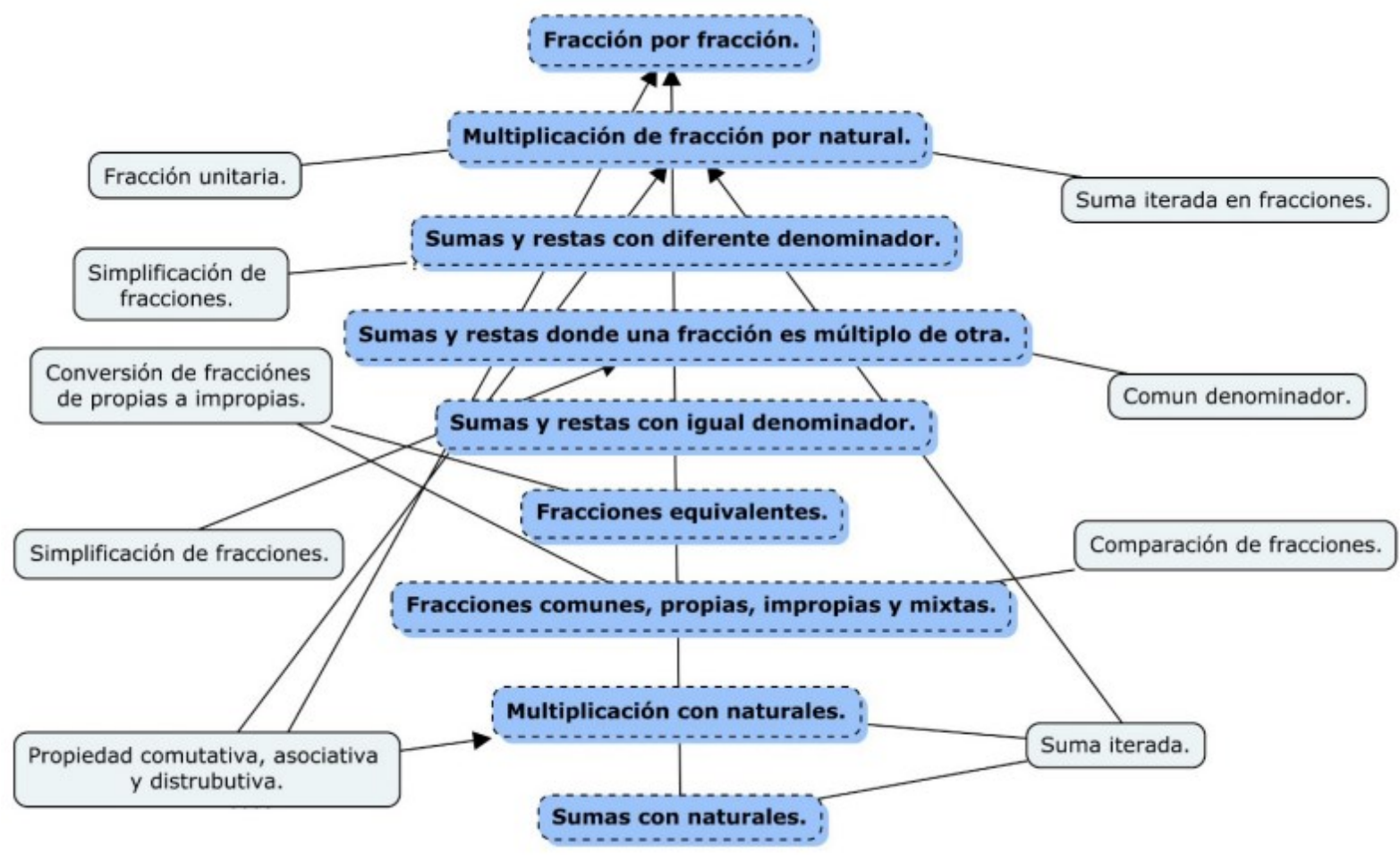

Figura 3. Exemplo de conexões entre conhecimentos de frações e operações (México)

Fonte: Lizarde (2019, p. 163).

Também há a possibilidade de abordar formativamente aspectos do subdomínio da prática matemática (KPM), cujos conhecimentos são necessários quando se adota estratégias de ensino com autonomia dos alunos para fazer matemática (PAENZA, 2009), ou seja, achar padrões e regularidades que permitem resolver problemas e construir constructos matemáticos. Nestes casos, o professor necessitará de conhecimento relativo à prática de definir e de demonstrar (CANO; FLORES, 2019) para analisar soluções dos alunos e orientar sobre sua abrangência, suficiência e adequação. Exemplos deste tipo de situação de prática estão na literatura e podem ser utilizadas em ação formativa, dentre as quais destacamos a identificação de padrões em soluções de alunos e análise de condições necessárias e suficientes para que tais processos forneçam resultados corretos (MORIEL JUNIOR et al., 2017).

Também inclui-se analisar matematicamente em que circunstâncias um erro comum de alunos - em que a soma de numeradores e denominadores entre si $\frac{a}{b} \oplus \frac{c}{d}=\frac{a+c}{b+d}$ - é válido (LOPES, 2008). Em seguida, pode-se explorar o algoritmo similar para dividir frações 
corretamente $\left(\frac{a}{b} \div \frac{c}{d}=\frac{a \div c}{b \div d}\right)$, discutindo sua validade do ponto de vista matemático (KPM), as características do resultado deste procedimento (KoT) e suas limitações/potencialidades didáticas durante o ensino (KMT) para responder a esta forma comum de alunos lidarem com tal conteúdo (KFLM). Isso demonstra que há uma conexão intensa entre subdomínios matemáticos e didáticos a ser trabalhada em formação docente.

\section{CONSIDERAÇÕES}

Neste trabalho discutimos teoricamente como a combinação das dimensões do MTSK com resultados científicos em Educação Matemática pode oferecer caminhos formativos para potencializar o desenvolvimento de conhecimento especializado docente sobre frações e operações, seu ensino e aprendizagem. Realizamos uma análise reflexiva a posteriori sobre dados empíricos de um cenário relativamente comum na formação docente (sujeito com lacuna ou limitação de conhecimento docente para lidar com uma questão de prática docente relevante). Foi possível avançar na identificação de possibilidades formativas hipotéticas fundamentadas para desenvolver todos os subdomínios do conhecimento especializado a partir de avanços científicos (conforme as referências bibliográficas citadas na seção anterior). Dentre os resultados obtidos, destacamos:

- recomendações de encadeamento de conceitos sobre frações e operações provenientes de especificações curriculares, pesquisas científicas ou opinião de professores experientes (KMLS), elaboradas levando em consideração as características de aprendizagem de estudantes, erros comuns e suas fontes prováveis, incluídos no KFLM;

- de conceitos, algoritmos, procedimentos, justificativas e fenômeno ligados a frações e suas operações (KoT), bem como, estabelecer diferentes conexões entre tais conceitos, pertencentes ao KSM;

- de explicações instrucionais e estratégias didáticas (KMT) que permitam ensinar para a compreensão por meio do exercício do fazer matemática, ao invés de apenas memorizá-la, incluindo conhecimentos da prática de definir e demonstrar (KPM).

Estes resultados demonstram o potencial do MTSK na formação docente em consonância com estudos antecedentes. Mas trazem novos elementos sobre a promoção do desenvolvimento docente em todas e em cada uma das dimensões do conhecimento especializado referente ao conteúdo frações e suas operações. Eles podem inspirar e servir de 
diretriz para que sejam criados caminhos formativos para outros conteúdos. Entretanto, nossos resultados não esgotam todo o conhecimento necessário a um docente para ensinar e fazer aprender o conteúdo em questão.

Parece razoável que nosso estudo possa interessar a formadores de professores em potencializar suas ações formativas, gestores interessados em (re)formular suas políticas ou projetos de curso de preparação inicial ou continuada e a pesquisadores na área. Isto ganha relevância haja vista que a literatura tem relatado existência significativa de lacunas e limitações de conhecimento docente em relação a aspectos elementares do conteúdo, seu ensino ou aprendizagem aqui discutidos. Os resultados podem ter as aplicações práticas na (re)construção de planos de formação docente, de projetos de curso de licenciatura ou formação continuada, de oficinas formativas e de produtos para o mesmo fim, como livros, podcasts, vídeos, plataformas digitais, aplicativos, dentre outros. Esses entendimentos tem respaldo de estudos antecedentes que algumas dessas ações já foram colocadas em prática pelos grupos mencionados (GARCIA; PRIETO; CATALÁN, 2017; QUIROGA; GAMBOA, 2017).

Uma limitação deste trabalho é que se trata de uma primeira aproximação ao tema, de forma teórico-analítica. Neste sentido, outros trabalhos são necessários para colocar em prática os caminhos formativos aqui discutidos para compreender os tempos, espaços, materiais necessários, impactos e limitações inerentes. Convidamos outros pesquisadores a colocarem em prática e descreverem os resultados para que seja possível também analisar a influência das diferentes variáveis de cada contexto e compreender seu potencial de extensão.

\section{REFERÊNCIAS}

AGUILAR, A. El conocimiento especializado de una maestra sobre la clasificación de las figuras planas. Un estudio de caso. 2016. 220 p. Tese de doutorado (Doutorado em Educação Matemática). Universidad de Huelva, Huelva.

ANGELO, C. L.; DOS SANTOS, J. R. V.; MELÃO, W. S. Licenciandos em Matemática e Situações da Matemática Escolar: um Estudo Exploratório sobre a Formação Inicial de Professores de Matemática. Revista Brasileira de Ensino de Ciência e Tecnologia, v. 2, n. 3, p. 41-59, 2009. Disponível em:

http://revistas.utfpr.edu.br/pg/index.php/rbect/article/view/552. Acesso em: 20 Dez. 2020.

ASHLOCK, R. B. Error patterns in computation: Using error patterns to improve instruction. Upper Saddle River, N.J.: Pearson Education, 2006.

BALL, D. L.; THAMES, M. H.; PHELPS, G. Content Knowledge for Teaching: What Makes It Special? Journal of teacher education, v. 59, n. 5, p. 389-407, 2008. 
BARBOSA, E. P. Os Por Quês Matemáticos dos Alunos na Formação dos Professores. In: XIII Conferência Interamericana de Educação Matemática, 13., 2011, Recife. Anais [...]. Recife, 2011. p. 1-12. Disponível em:

http://www.cimm.ucr.ac.cr/ocs/files/conferences/1/schedConfs/1/papers/611/public/6119763-1-PB.pdf. Acesso em: 20 Dez. 2020.

BAYOUD, J. M. A comparison of pre-service and experienced elementary teachers' pedagogical content knowledge (PCK) of common error patterns in fractions. 2011. 180 p. Dissertação (Mestrado em Artes e Educação). Departament of Education, American University of Beirut, Beirut.

BEHR, M. J.; LESH, R.; POST, T. R.; SILVER, E. A. Rational number concepts. In: LESH, R. e LANDAU, M. (Ed.). Acquisition of mathematics concepts and processes. New York: Academic Press, 1983. p. 91-125.

BERTONI, N. E. A construção do conhecimento sobre número fracionário. Bolema: Boletim de Educação Matemática, v. 21, n. 31, p. 209-237, 2008. Disponível em:

http://www.periodicos.rc.biblioteca.unesp.br/index.php/bolema/article/view/2111. Acesso em: 20 Dez. 2020.

BERTONI, N. E. Educação e linguagem matemática: Frações e números fracionários. Curitiba: PEDEad, 2009. 95 p. Disponível em: http://www.sbembrasil.org.br/files/fracoes.pdf. Acesso em: 14 Ago. 2013.

BOGDAN, R.; BIKLEN, S. Investigação qualitativa em educação: uma introdução à teoria e aos métodos. Porto: Porto Editora, 1991.

BRASIL. Base Nacional Comum Curricular (BNCC). Brasília: MEC, 2018. 595 p. Disponível em:

http://basenacionalcomum.mec.gov.br/images/BNCC_EI_EF 110518 versaofinal_site.pdf. Acesso em: 20 Dez. 2020.

CAMPOS, T. M. M.; SILVA, A. D. F. G. Conhecimento profissional docente de professoras das séries iniciais da educação básica acerca da equivalência de números racionais na representação fracionária em um processo de formação continuada. Revemat: revista eletrônica de educação matemática, v. 4, n. 1, p. 114-127, 2009.

CANO, M. C.; FLORES, E. Prácticas matemáticas: un avance en su caracterización. In: IV Congreso Iberoamericano sobre Conocimiento Especializado del Profesor de Matemáticas 2019, Huelva. Anais [...]. Huelva, 2019. p. 87-94. Disponível em:

http://www.redmtsk.com/wp-content/uploads/Actas-IV-CIMTSK-prueba-FINAL-290720.pdf. Acesso em: 20 Dez. 2020.

CARRILlO, J.; CLIMENT, N.; CONTRERAS, L. C.; MONTES, M. Á.; ESCUDERO, D.; MEDRANO, E. F. Un marco teórico para el Conocimiento especializado del Profesor de Matemáticas. Huelva: Universidad de Huelva Publicaciones, 2014. 93 p.

CARRILlO, J.; CONTRERAS, L. C.; CLIMENT, N.; MONTES, M.; ESCUDERO, D. I.; FLORES, E.; CATALÁN, M. C. M. Didáctica de las Matemáticas para maestros de Educación Primária. Madri: Paraninfo, 2016. 357 p. 
CARRILlO, J.; MONTES, M. A.; CONTRERAS, L. C.; CLIMENT, N. Les connaissances du professeur dans une perspective basée sur leur spécialisation: MTSK. Annales de Didactique et de Sciences Cognitivess, v. 22, n. 1, p. 185-205, 2017. Disponível em: https://goo.gl/Ly1ekK. Acesso em: 20 Dez. 2020.

CARRILLO-YAÑEZ, J.; CLIMENT, N.; MONTES, M.; CONTRERAS, L. C.; FLORESMEDRANO, E.; ESCUDERO-ÁVILA, D.; VASCO, D.; ROJAS, N.; FLORES, P.; AGUILAR-GONZÁLEZ, Á.; RIBEIRO, M.; MUÑOZ-CATALÁN, M. C. The mathematics teacher's specialised knowledge (MTSK) model. Research in Mathematics Education, v. 20, n. 3, p. 236-253, 2018. Disponível em: https://doi.org/10.1080/14794802.2018.1479981. Acesso em: 20 Dez. 2020.

CASTRO, R. A.; OLIVEIRA, N. Número fracionário: estudo histórico, epistemológico e da transposição didática. Revista de educação, v. 12, n. 13, p. 59-70, 2009. Disponível em: http://www.sare.unianhanguera.edu.br/index.php/reduc/article/viewFile/824/664. Acesso em: 20 Dez. 2020.

CATALÁN, M. C. M.; CARRILLO, J. Didáctica de las Matemáticas para maestros de Educación Infantil. Madri: Paraninfo, 2018. 318 p.

CHARALAMBOUS, C. Y.; HILL, H. C.; BALL, D. L. Prospective teachers' learning to provide instructional explanations: how does it look and what might it take? Journal of Mathematics Teacher Education, n. 14, p. 441-463, 2011.

CLIMENT, N.; MONTES, M. Diseño de tareas para la formación de profesores de matemáticas a partir de MTSK. In: IV Congreso Iberoamericano sobre Conocimiento Especializado del Profesor de Matemáticas 2019, Huelva. Anais [...]. Huelva, 2019. p. 60-70. Disponível em: http://www.redmtsk.com/wp-content/uploads/Actas-IV-CIMTSK-pruebaFINAL-290720.pdf. Acesso em: 20 Dez. 2020.

CONTRERAS, L. C.; MONTES, M. A.; CLIMENT, N.; CARRILLO, J. Introducción al modelo MTSK: origen e investigaciones realizadas. For-Mate, v. 1, n. 1, p. 1-10, 2017. Disponível em: https://www.researchgate.net/publication/313824049. Acesso em: 20 Dez. 2020.

DRECHMER, P. A. D. O.; ANDRADE, S. V. R. D. O estudo de frações e seus cinco significados. In: CONFERÊNCIA INTERAMERICANA DE EDUCAÇÃO MATEMÁTICA - CIAEM, 13., 2011, Recife. Anais [...]. Recife, 2011. p. 1-8. Disponível em: http://www.diaadiaeducacao.pr.gov.br/portals/pde/arquivos/1959-8.pdf ou

http://cimm.ucr.ac.cr/ocs/index.php/xiii_ciaem/xiii_ciaem/paper/viewFile/1660/728. Acesso em: 20 Dez. 2020.

FÁVERO, M. H.; PINA NEVES, R. D. S. A divisão e os racionais: revisão bibliográfica e análise. Zetetiké, v. 20, n. 1, p. 39-78, 2012. Disponível em: http://www.fae.unicamp.br/zetetike/viewarticle.php?id=553. Acesso em: 20 Dez. 2020. 
FERREIRA, E. S. Onze avos, doze avos,... De onde vem este termo avo? Revista Brasileira de História da Matemática, v. 6, n. 11, p. 97-108, 2006. Disponível em: http://goo.gl/TCDyGJ. Acesso em: 20 Dez. 2020.

FIORENTINI, D. A formação Matemática e didático-pedagógica nas disciplinas da Licenciatura em Matemática. Revista de Educação, n. 18, p. 107-115, 2005.

FORRESTER, T.; CHINNAPPAN, M. The predominance of procedural knowledge in fractions. Shaping the future of mathematics education, p. 185-192, 2010.

GARCIA, M.; PRIETO, N.; CATALÁN, M. Una propuesta colaborativa para enriquecer la formación matemática inicial y continua de maestros de infantil. In: III Jornadas de Investigación en Didáctica de las Matemáticas, 3., 2017, Huelva. Anais [...]. Huelva, 2017. p. 102-107. Disponível em: http:/www.redmtsk.com/wp-content/uploads/Actas-III-JornadasSIDM-2017.pdf. Acesso em: 20 Dez. 2020.

GUERRA, R. B.; SILVA, F. H. S. D. As Operações com Frações e o Princípio da Contagem. Bolema: Boletim de Educação Matemática, v. 21, n. 31, p. 41-54, 2008. Disponível em: http://www.redalyc.org/pdf/2912/291221883004.pdf. Acesso em: 20 Dez. 2020.

INEP. Banco de dados do SAEB de 1995 a 2005. Brasília: MEC, 2014. Disponível em: http://download.inep.gov.br/educacao_basica/prova_brasil_saeb/resultados/matematicauf(urb_s_fed)95-05-8a.xls. Acesso em: 16 ago 2014.

INEP. Censo da Educação Básica 2016: notas estatísticas. Brasília: MEC, p. 29, 2017. Disponível em:

http://download.inep.gov.br/educacao basica/censo_escolar/notas_estatisticas/2017/notas est atisticas_censo_escolar_da_educacao_basica_2016.pdf. Acesso em: 20 Dez. 2020.

KILPATRICK, J.; SPANGLER, D. A. Educating Future Mathematics Education Professors. In: ENGLISH, L. D. e KIRSHNER, D. (Ed.). Handbook of International Research in Mathematics Education. New York: Routledge, 2015. p. 297-310.

KILPATRICK, J.; SWAFFORD, J.; FINDELL, B. Adding it up: Helping children learn mathematics. Washington, DC: National Academies Press, 2001. Disponível em: http://www.sjsd.k12.mo.us/cms/lib3/MO01001773/Centricity/Domain/872/Adding\%20it\%20 Up.pdf. Acesso em: 20 Dez. 2020.

LIMA, E. L. Divisão de números racionais escritos na forma de frações. Revista do Professor de Matemática, v. 3, p. 40-42, 1983.

LIN, C.-Y.; BECKER, J.; BYUN, M.-R.; YANG, D.-C.; HUANG, T.-W. Preservice Teachers' Conceptual and Procedural Knowledge of Fraction Operations: A Comparative Study of the United States and Taiwan. School Science and Mathematics, v. 113, n. 1, p. 41 51, 2013. Disponível em: http://dx.doi.org/10.1111/j.1949-8594.2012.00173.x. Acesso em: 20 Dez. 2020.

LIZARDE, E. MTSK y formación docente continua: explorando horizontes de posibilidades. In: IV Congreso Iberoamericano sobre Conocimiento Especializado del Profesor de Matemáticas 2019, Huelva. Anais [...]. Huelva, 2019. p. 158-166. Disponível em: 
http://www.redmtsk.com/wp-content/uploads/Actas-IV-CIMTSK-prueba-FINAL-290720.pdf. Acesso em: 20 Dez. 2020.

LOPES, A. J. O que nossos alunos podem estar deixando de aprender sobre frações, quando tentamos lhes ensinar frações. Bolema: Boletim de Educação Matemática, v. 21, n. 31, p. 122, 2008. Disponível em: http://www.periodicos.rc.biblioteca.unesp.br/index.php/bolema/issue/view/756. Acesso em: 20 Dez. 2020.

MA, L. Knowing and teaching elementary mathematics: Teachers' understanding of fundamental mathematics in China and the United States. Lawrence Erlbaum Associates Mahwah, NJ, 1999. 198 p

MONTES, M. A.; CONTRERAS, L. C.; CARRILLO, J. Conocimiento del profesor de matemáticas: Enfoques del MKT y del MTSK. In: Investigación en Educación Matemática XVII, 17., 2013, Bilbao, Espanha. Anais [...]. Bilbao, Espanha, 2013. p. 403-410. Disponível em: http://funes.uniandes.edu.co/12056/1/Montes2014Conocimiento.pdf. Acesso em: 20 Dez. 2020 .

MOREIRA, P. C.; DAVID, M. M. M. S. Matemática escolar, matemática científica, saber docente. Zetetiké, v. 11, n. 19, p. 57-80, 2003. Disponível em:

http://www.fae.unicamp.br/revista/index.php/zetetike/article/view/2490/2250. Acesso em: 20 Dez. 2020.

MOREIRA, P. C.; FERREIRA, M. C. C. A Teoria dos Subconstrutos e o Número Racional como Operador: das estruturas algébricas às cognitivas. Bolema: Boletim de Educação Matemática, v. 21, n. 31, p. 103-127, 2008. Disponível em: http://www.redalyc.org/pdf/2912/291221883007.pdf. Acesso em: 20 Dez. 2020.

MORIEL JUNIOR, J. G. Conhecimento especializado para ensinar divisão de frações. 2014. 162 p. Tese (Doutorado em Educação em Ciências e Matemática). PPGECEM/REAMEC, Universidade Federal de Mato Grosso, Cuiabá.

MORIEL JUNIOR, J. G. Uma nova caracterização para as interpretações de divisão de frações. In: VIII Congreso Iberoamericano de Educación Matemática: Libro de actas, 8., 2017, Madri. Anais [....]. Madri, 2017. p. 588-596. Disponível em: http://cibem.org/images/site/LibroActasCIBEM/ComunicacionesLibroActas_CB801-900.pdf. Acesso em: 20 Dez. 2020.

MORIEL JUNIOR, J. G.; DUARTE, E. B. Mapeamento global da produção sobre Mathematics Teacher's Specialized Knowledge no Google Scholar até 2019. Research, Society and Development, v. 9, n. 11, p. e71191110526, 12/02 2020. Disponível em: https://rsdjournal.org/index.php/rsd/article/view/10526. Acesso em: 20 Dez. 2020.

MORIEL JUNIOR, J. G.; TEIXEIRA, M. C.; SILVA FILHO, V. P.; ZIMMERMANN JUNIOR, C. Questões para potencializar o conhecimento especializado para ensinar divisão de frações. In: CIEM, 2017, Canoas. Anais [...]. Canoas, 2017. p. 1-12. Disponível em: http://www.conferencias.ulbra.br/index.php/ciem/vii/paper/viewFile/7289/3446. Acesso em: 20 Dez. 2020. 
MORIEL JUNIOR, J. G.; WIELEWSKI, G. D. Por quês matemáticos na Revista do Professor de Matemática. Revista de Educação Pública, v. 22, n. 51, p. 975-998, 2013a. Disponível em: http://200.129.241.78/ojs/index.php/educacaopublica/article/view/1266/1018. Acesso em: 20 Dez. 2020.

MORIEL JUNIOR, J. G.; WIELEWSKI, G. D. Seis possibilidades de resposta para o por quê matemático sobre divisão de frações. In: Seminário Educação - SEMIEDU, 2013, Cuiabá. Anais [...]. Cuiabá, 2013b. p. 1 - 15. Disponível em: http://goo.g1/LuAiYI. Acesso em: 20 Dez. 2020.

MORIEL JUNIOR, J. G.; WIELEWSKI, G. D. Base de conhecimento de professores de matemática: do genérico ao especializado. Revista de Ensino, Educação e Ciências Humanas, v. 18, n. 2, p. 126-133, 2017. Disponível em: https://revista.pgsskroton.com/index.php/ensino/article/view/4579/3572. Acesso em: 20 Dez. 2020.

MORIEL JUNIOR, J. G.; WIELEWSKI, G. D.; CARRILLO, J. Meta-análise sobre conhecimento para ensinar divisão de frações. Bolema: Boletim de Educação Matemática, v. 33, n. 65, p. 988-1026, 2019. Disponível em: http://dx.doi.org/10.1590/1980$4415 \mathrm{v} 33 \mathrm{n} 65 \mathrm{a} 02$

http://www.scielo.br/pdf/bolema/v33n65/1980-4415-bolema-33-65-0988.pdf. Acesso em: 20 Dez. 2020.

NAISER, E. A.; WRIGHT, W. E.; CAPRARO, R. M. Teaching fractions: Strategies used for teaching fractions to middle grades students. Journal of research in childhood education, $\mathrm{v}$. 18, n. 3, p. 193-198, 2003.

NUNES, T.; BRYANT, P. Crianças fazendo matemática. Porto Alegre: Artes Médicas, 1997.

PAENZA, A. Matemática, cadê você?: sobre números, personagens, problemas e curiosidades. Rio de Janeiro: Civilização Brasileira, 2009.

PETIT, M. M.; LAIRD, R. E.; MARSDEN, E. A focus on fractions: bringing research to the classroom. New York \& London: Routledge, 2010.

PINILLA, M. I. F. Fractions: conceptual and didactic aspects. Acta Didactica Universitatis Comenianae, n. 7, p. 81-115, 2007. Disponível em:

http://www.ddm.fmph.uniba.sk/ADUC/files/Issue7/05Pinilla.pdf. Acesso em: 20 Dez. 2020.

QUIROGA, R.; GAMBOA, M. Contribución del MTSK em la Elaboración del Plan de Formación de Profesores de Matemática. In: III Jornadas de Investigación en Didáctica de las Matemáticas, 3., 2017, Huelva. Anais [...]. Huelva, 2017. p. 125-130. Disponível em: http://www.redmtsk.com/wp-content/uploads/Actas-III-Jornadas-SIDM-2017.pdf. Acesso em: 20 Dez. 2020.

RIBEIRO, M. Tareas para alumnos y tareas para la formación: discutiendo el conocimiento especializado del profesor y del formador de profesores de matemáticas. In: XX Jornadas Nacionales de Educación Matemática, 2016, Valparaíso. Anais [...]. Valparaíso, 2016. p. 31- 
39. Disponível em: http://static.ima.ucv.cl.s3.amazonaws.com/wpcontent/uploads/2016/03/Acta-XXJNEM-final.pdf. Acesso em: 20 Dez. 2020.

RICONSCENTE, M. Mobile learning game improves 5th graders' fractions knowledge and attitudes. GameDesk Institute, p. 1-44, 2011.

ROJAS, N. Caracterización del conocimiento especializado del profesor de matemáticas: Un estudio de casos. 2014. 200p. Tese (Doutorado em Educação Matemática). Universidad de Granada, Granada.

ROWLAND, T. The Knowledge Quartet: The Genesis and Application of a Framework for Analysing Mathematics Teaching and Deepening Teachers' Mathematics Knowledge.

SISYPHUS - Journal of education, v. 1, n. 3, p. 15-43, 2013. Disponível em: http://revistas.rcaap.pt/sisyphus/article/view/3705/2859. Acesso em: 20 Dez. 2020.

SANTOS, R. S. D.; SANTOS, M. C. D.; CAMPOS, T. M. M. Estratégias utilizadas pelos alunos da educação básica na resolução de questões sobre números racionais na prova do saepe/sistema de avaliação educacional de Pernambuco. In: ENCONTRO NACIONAL DE EDUCAÇÃO MATEMÁTICA - ENEM, 11., 2013, Curitiba. Anais [...]. Curitiba, 2013. p. 115. Disponível em: http://sbem.esquiro.kinghost.net/anais/XIENEM/pdf/1500_1265_ID.pdf. Acesso em: 20 Dez. 2020.

SHULMAN, L. S. Those who understand: Knowledge growth in teaching. Educational researcher, v. 15, n. 2, p. 4-14, 1986. Disponível em: http:/www.itp.wceruw.org/documents/Shulman 1986.pdf. Acesso em: 20 Dez. 2020.

SILVA, M. J. F.; ALMOULOUD, S. A. As Operações com Números Racionais e seus Significados a partir da Concepção Parte-todo. Bolema: Boletim de Educação Matemática, v. 21, n. 31, p. 55-78, 2008. Disponível em: http://www.leoakio.com/wa files/2105-8989-2PB.pdf. Acesso em: 20 Dez. 2020.

TARDIF, M. Saberes docentes e formação profissional. Petrópolis: Vozes, 2002.

VASCO, D.; MORIEL JUNIOR, J. G.; CONTRERAS, L. C. Subdominios KoT y KSM del Mathematics Teacher's Specialised Knowledge (MTSK): definición, categorías y ejemplos. In: III Jornadas de Investigación en Didáctica de las Matemáticas, 3., 2017, Huelva. Anais [...]. Huelva, 2017. p. 29-37. Disponível em: http://www.redmtsk.com/wpcontent/uploads/Actas-III-Jornadas-SIDM-2017.pdf. Acesso em: 20 Dez. 2020.

WU, H.-H. Teaching Fractions According to the Common Core Standards. American Mathematical Society, p. 1-88, 2011.

\section{NOTAS}

\section{AGRADECIMENTOS}

Os autores agradecem a Coordenação de Aperfeiçoamento de Pessoal de Nível Superior - Brasil (CAPES) pelo apoio ao Programa de Mestrado em Ensino do IFMT e pelo Portal de Periódicos. 


\section{FINANCIAMENTO}

Fomento da FAPEMAT (Edital Universal 42/2016/FAPEMAT) e do IFMT (Chamada 001/2019 DPG/PROPES/IFMT e Edital 31/2020 DPIT/PROPES/IFMT); Coordenação de Aperfeiçoamento de Pessoal de Nível Superior - Brasil (CAPES) - Código de Financiamento 001.

\section{CONTRIBUIÇÕES DE AUTORIA}

Resumo/Abstract/Resumen: Jeferson Gomes Moriel Junior e Gladys Denise Wielewski Introdução: Jeferson Gomes Moriel Junior e Gladys Denise Wielewski Referencial teórico: Jeferson Gomes Moriel Junior e Gladys Denise Wielewski Análise de dados: Jeferson Gomes Moriel Junior e Gladys Denise Wielewski Discussão dos resultados: Jeferson Gomes Moriel Junior e Gladys Denise Wielewski Conclusão e considerações finais: Jeferson Gomes Moriel Junior e Gladys Denise Wielewski Referências: Jeferson Gomes Moriel Junior e Gladys Denise Wielewski Revisão do manuscrito: Jeferson Gomes Moriel Junior e Gladys Denise Wielewski Aprovação da versão final publicada: Jeferson Gomes Moriel Junior e Gladys Denise Wielewski

\section{CONFLITOS DE INTERESSE}

Os autores declaram não haver nenhum conflito de interesse de ordem pessoal, comercial, acadêmico, político e financeiro referente a este manuscrito.

\section{DISPONILIDADE DE DADOS DE PESQUISA}

Os dados que suportam os resultados deste estudo poderão ser disponibilizados mediante solicitação plausível, cabendo aos autores determinar a plausibilidade da solicitação, bem como as condições (licença) de acesso e uso.

\section{CONSENTIMENTO DE USO DE IMAGEM}

Não se aplica

\section{APROVAÇÃO DO COMITÊ DE ÉTICA EM PESQUISA}

Não se aplica

\section{COMO CITAR - ABNT}

JUNIOR, Jeferson Gomes Moriel; WIELEWSKI, Gladys Denise. Potenciais oportunidades formativas com MTSK e pesquisas científicas sobre frações e operações. REAMEC - Rede Amazônica de Educação em Ciências e Matemática. Cuiabá, v. 9, n.1, janeiro-abril, 2021. DOI: 10.26571/reamec.v9i1.11462.

\section{COMO CITAR - APA}

Junior, J. G. M.; Wielewski, G. D. Potenciais oportunidades formativas com MTSK e pesquisas científicas sobre frações e operações. REAMEC - Rede Amazônica de Educação em Ciências e Matemática. Cuiabá, v. 9, n.1, janeiro-abril, 2021. DOI: 10.26571/reamec.v9i1.11462.

\section{LICENÇA DE USO}

Licenciado sob a Licença Creative Commons Attribution-NonCommercial 4.0 International (CC BY-NC 4.0). Esta licença permite compartilhar, copiar, redistribuir o manuscrito em qualquer meio ou formato. Além disso, permite adaptar, remixar, transformar e construir sobre o material, desde que seja atribuído o devido crédito de autoria e publicação inicial neste periódico.

\section{DIREITOS AUTORAIS}

Os direitos autorais são mantidos pelos autores, os quais concedem à Revista REAMEC - Rede Amazônica de Educação em Ciências e Matemática - os direitos exclusivos de primeira publicação. Os autores não serão remunerados pela publicação de trabalhos neste periódico. Os autores têm autorização para assumir contratos adicionais separadamente, para distribuição não exclusiva da versão do trabalho publicada neste periódico (ex.: publicar em repositório institucional, em site pessoal, publicar uma tradução, ou como capítulo de livro), com reconhecimento de autoria e publicação inicial neste periódico. Os editores da Revista têm o direito de proceder a ajustes textuais e de adequação às normas da publicação.

\section{PUBLISHER}

Universidade Federal de Mato Grosso. Programa de Pós-graduação em Educação em Ciências e Matemática (PPGECEM) da Rede Amazônica de Educação em Ciências e Matemática (REAMEC). Publicação no Portal de Periódicos UFMT. As ideias expressadas neste artigo são de responsabilidade de seus autores, não representando, necessariamente, a opinião dos editores ou da referida universidade. 


\section{EDITOR}

Dailson Evangelista Costa (D)

\section{HISTÓRICO}

Submetido: 26 de novembro de 2020 .

Aprovado: 04 de janeiro de 2021.

Publicado: 26 de janeiro de 2021. 